\title{
Characterization of Nitrotyrosine-Modified Proteins in Cerebrospinal Fluid
}

\author{
Ashley S. Beasley • Caroline Anderson • \\ Justin McArthur • Ned Sacktor • Avindra Nath • \\ Robert J. Cotter
}

Published online: 11 March 2010

(C) Springer Science+Business Media, LLC 2010

\begin{abstract}
Background HIV-associated neurocognitive disorders (HAND) has been associated with the up-regulation of various oxidative stress pathways. Previous studies have linked the neuronal damage observed in individuals diagnosed with HAND to increased nitrotyrosine modification of neuronal proteins.

Materials and methods Tyrosine nitration alters protein structure and function, affects biological half-life, and potentially prevents the phosphorylation of key tyrosine residues involved in signal transduction pathways. Therefore, in this study we employed proteomics-based experimental approaches to investigate nitrotyrosine-modified proteins in pooled cerebrospinal fluid (CSF) of individuals diagnosed with HAND. To identify specific nitrotyrosine-modified proteins in the CSF of individuals diagnosed with HAND, affinity purification and high-performance tandem mass spectrometry are utilized in a "bottom-up" proteomics approach.
\end{abstract}

Electronic supplementary material The online version of this article (doi:10.1007/s12014-010-9041-4) contains supplementary material, which is available to authorized users.

\footnotetext{
A. S. Beasley $\cdot$ R. J. Cotter $(\bowtie)$

Pharmacology and Molecular Sciences,

Johns Hopkins University School of Medicine,

725 North Wolfe Street, Biophysics Building, B7,

Baltimore, MD 21205, USA

e-mail: rcotter@jhmi.edu

C. Anderson $\cdot$ J. McArthur $\cdot$ N. Sacktor $\cdot$ A. Nath Neurology, Johns Hopkins University School of Medicine, 725 North Wolfe Street, Biophysics Building, B7, Baltimore, MD 21205, USA

A. Nath

Neuroscience, Johns Hopkins University School of Medicine, 725 North Wolfe Street, Biophysics Building, B7,

Baltimore, MD 21205, USA
}

Results From tandem mass spectrometric analysis, we identified major proteins that underwent nitration as a result of nitro-oxidative stress in the CSF of individuals diagnosed with HAND. We also utilized analytical and biochemical techniques to characterize the expression and modification site of in vivo nitrated lipocalin-type prostaglandin-D synthase in HAND CSF.

Keywords BSA · CSF · HIV · HAND · Oxidative stress · Tyrosine nitration

$\begin{array}{ll}\text { Abbreviations } \\ \text { BSA } & \text { Bovine Serum Albumin } \\ \text { CSF } & \text { Cerebrospinal fluid } \\ \text { HIV } & \text { Human Immunodeficiency Virus } \\ \text { HAND } & \text { HIV-associated neurocognitive disorders } \\ \text { L-PGDS } & \text { Lipocalin-type prostaglandin-D synthase } \\ \text { PTM }^{-} & \text {Post-translational modification } \\ \text { ONOO }^{-} & \text {Peroxynitrite }\end{array}$

\section{Introduction}

During the initial phases of human immunodeficiency virus (HIV) infection, the virus penetrates the blood-brain barrier and invades the host central nervous system (CNS). This leads to a cascade of intracellular anti-viral events including the production of oxidative stress that facilitate the control of viral replication and production [1]. The over production of these anti-viral responses have been linked to various neurological complications due to neuronal damage and toxicity, such as HIV-associated neurocognitive disorders (HAND) [1, 2]. However, with the advent of highly active antiretroviral therapy (HAART) the incidence and severity of HAND has drastically decreased but the prevalence of 
HAND has increased [3]. HAART has reduced the viral load, significantly improved the $\mathrm{CD} 4^{+} \mathrm{T}$-cell counts, and extended the life expectancy of individuals infected with HIV. Consequently, the life expectancy benefits associated with HAART and the low turnover of HIV-infected cells in the CNS has led to the progressive rise in milder HIVassociated cognitive disorders. Little is known about the mechanisms and pathways associated with the development and progression of HAND; therefore, current diagnosis of HAND requires extensive neuropsychological testing. Although the mechanism of development of HAND is unknown, previous studies of individuals diagnosed with HAND have shown a correlation between the degree of severity of HAND and elevated levels of inducible nitric oxide synthase (iNOS) [4]. The enzymatic activity of iNOS leads to the production of nitric oxide (NO), which can act as a cytotoxic effector molecule or a pathogenic mediator when produced at high rates [5]. Elevated levels of free reactive nitrogen species interact with neuronal proteins and lead to the selective nitration of tyrosine residues. Protein tyrosine nitration is a dynamic and selective posttranslational modification that is a well-established biomarker of oxidative stress. Nitrotyrosine is the product of the uncatalyzed reaction of a tyrosine residue and peroxynitrite $\left(\mathrm{ONOO}^{-}\right)$, which is a powerful oxidant produced from the reaction of $\mathrm{NO}$ and superoxide (Fig. 1). In addition to the uncatalyzed reaction, catalyzed reactions of $\mathrm{ONOO}^{-}$with $\mathrm{CO}_{2}$, transition metals, and myeloperoxidase can also lead to the formation of nitrotyrosine [6]. Tyrosine nitration alters protein structure and function, affects biological half-life, solubility, and increases the degree of protein degradation [5]. The addition of the bulky $-\mathrm{NO}_{2}$ group also imposes steric restrictions on the tyrosine aromatic ring which can lead to the inhibition of phosphorylation of the phenolic hydroxyl group of the phenolic ring

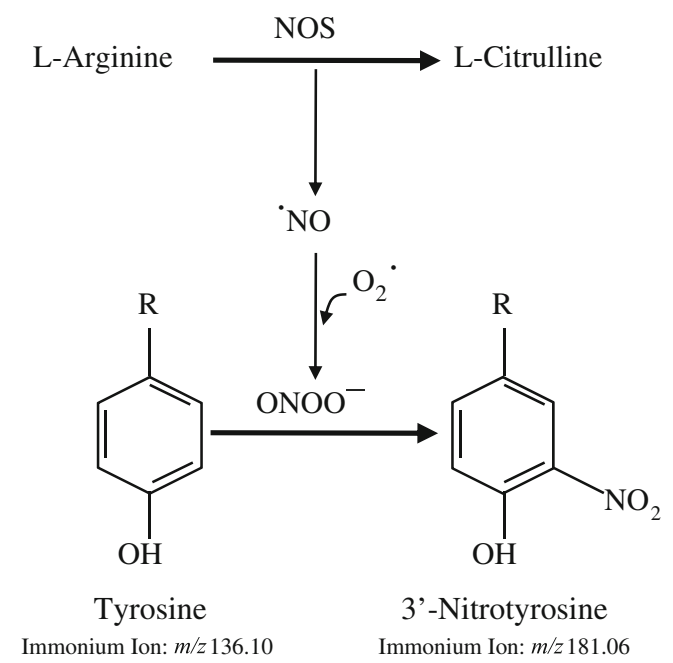

Fig. 1 Tyrosine nitration pathway of tyrosine [6]. Therefore, characterization of nitrotyrosinemodified proteins in cerebrospinal fluid (CSF) offers unique opportunity for the identification of key proteins that contribute to the HIV-induced effects of neurological dysfunction.

In this study, we employ affinity-based purification and high-performance tandem mass spectrometry in a "bottomup" proteomics approach to investigate nitrotyrosinemodified proteins in the CSF of individuals diagnosed with HAND.

\section{Materials and Methods}

In vitro nitration of $B S A$ and $L-P G D S$ One milligram of bovine serum albumin (BSA; Sigma, St. Louis, MO; and Sigma) was incubated with a $1.54 \mathrm{mM}(100 \mu \mathrm{L})$ solution of peroxynitrite (Upstate, Lake Placid, NY) in $0.3 \mathrm{M} \mathrm{NaOH}$ for $45 \mathrm{~min}$ at room temperature. Following nitration, the peroxynitrite-treated BSA ( $n$-BSA) solution was desalted with protein desalting spin columns (Pierce, Rockford, IL).

High-Performance Liquid Chromatography Analysis The desalting spin column flowthrough fraction was used to validate in vitro peroxynitrite-treated BSA. One hundred microliters of $n$-BSA $(100 \mu \mathrm{g})$ in $0.1 \%$ trifluoroacetic acid (TFA) was injected onto a $250 \times 2.0 \mathrm{~mm}$ Jupiter $5 \mu \mathrm{C}_{4}$ 300A (Phenomenex, Torrance, CA) reversed-phase column. The aqueous mobile phase A consisted of $0.1 \%$ TFA in water (Solvent A) and the organic mobile phase consisted of $0.1 \%$ TFA in ACN (Solvent B). The nitrated and nonnitrated protein was eluted with the following gradient: $30 \%$ Solvent B at $0-5 \mathrm{~min}, 50 \%$ Solvent B at $35 \mathrm{~min}$, $100 \%$ Solvent B at $40 \mathrm{~min}$, and 3\% Solvent B at $50 \mathrm{~min}$. The absorbance was monitored at both 214 and $350 \mathrm{~nm}$.

CSF Specimens The human CSF samples were obtained from non-HIV-infected and HAND diagnosed individuals from the Lumbar Puncture Clinic at Johns Hopkins Hospital. The samples were divided into two groups: nonHIV $(n=3)$ and HAND $(n=4)$. The non-HIV group consisted of two patients with normal pressure hydrocephalus and one with possible multiple sclerosis. None of them had any risk factors for HIV infection. The collected CSF samples were centrifuged at $3,000 \mathrm{rpm}$ for $10 \mathrm{~min}$ to remove cellular debris. The cell-free CSF supernatant was stored at $-80^{\circ} \mathrm{C}$ in $1.0 \mathrm{~mL}$ aliquots until further analysis to prevent sample degradation. Prior to analysis, the CSF samples were heated at $60^{\circ} \mathrm{C}$ for $20 \mathrm{~min}$ and pooled. Pooling of CSF was necessary since the detailed proteomic analysis required large amounts of CSF. CSF samples from ten individuals were initially screened for nitrotyrosine by slot blot as previously described [7] and the four HAND CSF samples with the highest levels of nitrotyrosine 
content were pooled for further analysis. This optimized our chances for detection of nitrotyrosine-modified proteins.

Nitrotyrosine Enrichment Prior to affinity purification of the nitrotyrosine-modified proteins, the control and diseased (HAND) CSF were immunodepleted with the Proteoseek $^{\mathrm{TM}}$ antibody-based albumin/IgG removal kit (Pierce, Rockford, IL). Following immunodepletion, the CSF samples were incubated with $100 \mu \mathrm{g}$ of antinitrotyrosine (clone 1A6) agarose conjugate (Upstate, Lake Placid, NY) overnight at $4{ }^{\circ} \mathrm{C}$ with gentle agitation to maintain optimal distribution of the antibody resin in the protein mixture. The resin was collected by centrifugation at $1,500 \times \mathrm{g}$ for $1 \mathrm{~min}$ and washed twice with phosphate buffer saline (PBS, $\mathrm{pH}$ 7.4). The nitrated proteins were eluted with $5 \%(v / v)$ formic acid in water and the fractions were concentrated in a speedvac.

Lipocalin-type Prostaglandin D Synthase Enrichment Co-immunoprecipitation of lipocalin-type prostaglandin D synthase (L-PGDS) was performed according to the Catch and Release v2.0 kit (Upstate, Lake Placid, NY) recommendations. The pooled albumin/IgG depleted CSF samples were incubated with the anti-LPGDS monoclonal antibody (Cayman Chemical, Ann Arbor, MI) at $4^{\circ} \mathrm{C}$ overnight. Following incubation, the spin column was washed twice with $1 \times$ wash buffer and each wash was centrifuged at 5,000 rpm for $1 \mathrm{~min}$. The antigen:antibody complex was eluted from the affinity resin with non-denaturing elution buffer (PBS, pH7.4) and the affinity purification fractions were concentrated in the speedvac and resuspended in $50 \mu \mathrm{L}$ of PBS (pH7.4). The above procedure was utilized to enrich the nitrotyrosine-modified protein from the LPGDS-enriched CSF samples.

Sodium dodecyl sulfate-polyacrylamide gel electrophoresis The immunoprecipitation fractions were loaded and separated by sodium dodecyl sulfate-polyacrylamide gel electrophoresis (SDS-PAGE) on a NuPAGE 4-12\% (w/v) Bis-Tris gel (Invitrogen, Carlsbad, CA) and visualized via silver staining $[8,9]$. Briefly, the gels were fixed in $50 \%(v / v)$ ethanol $(\mathrm{EtOH}) /$
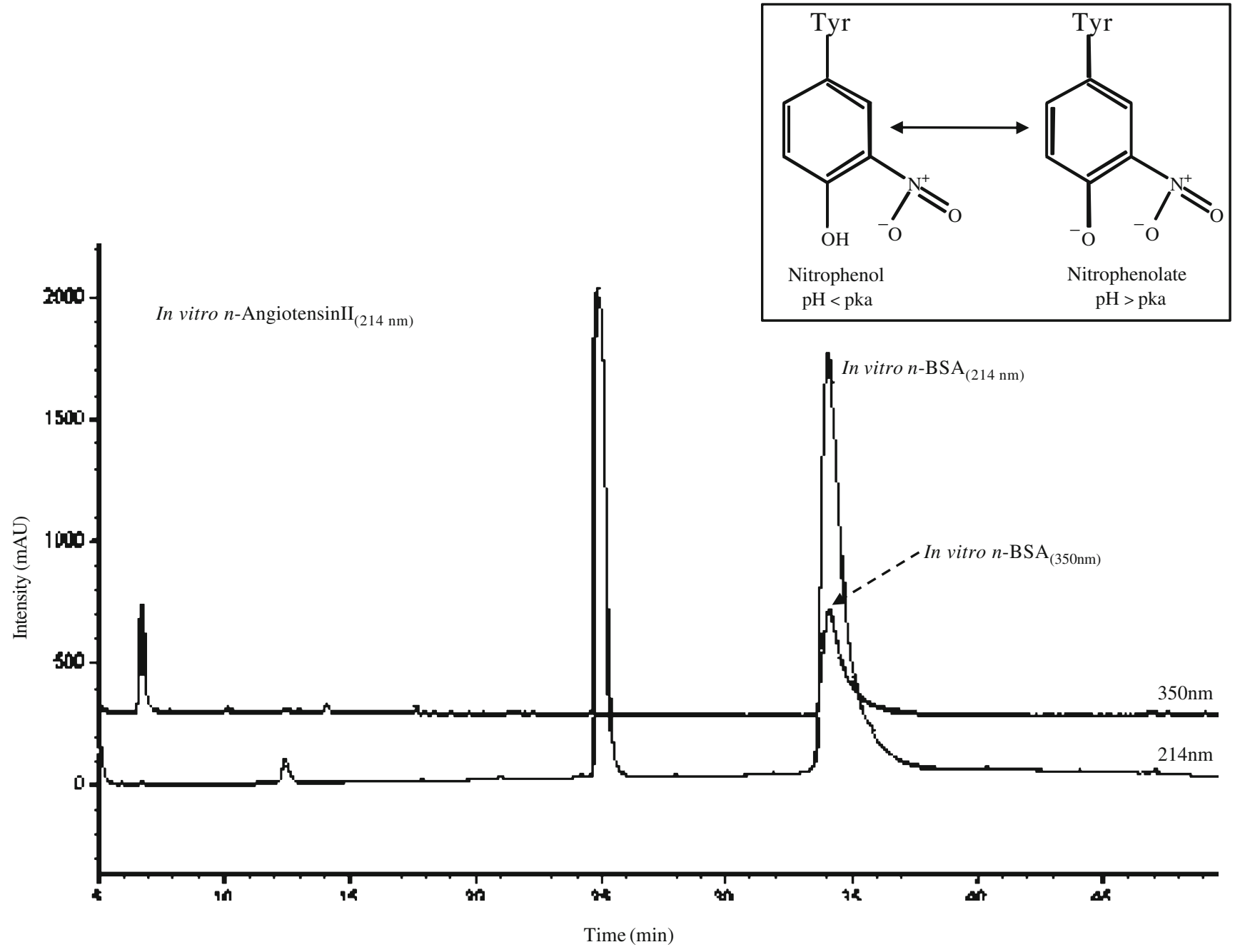

Fig. 2 Reversed-phase LC analysis of in vitro $n$-BSA containing unmodified Angiotensin II (internal non-nitrated standard) at 214 and $350 \mathrm{~nm}$ 


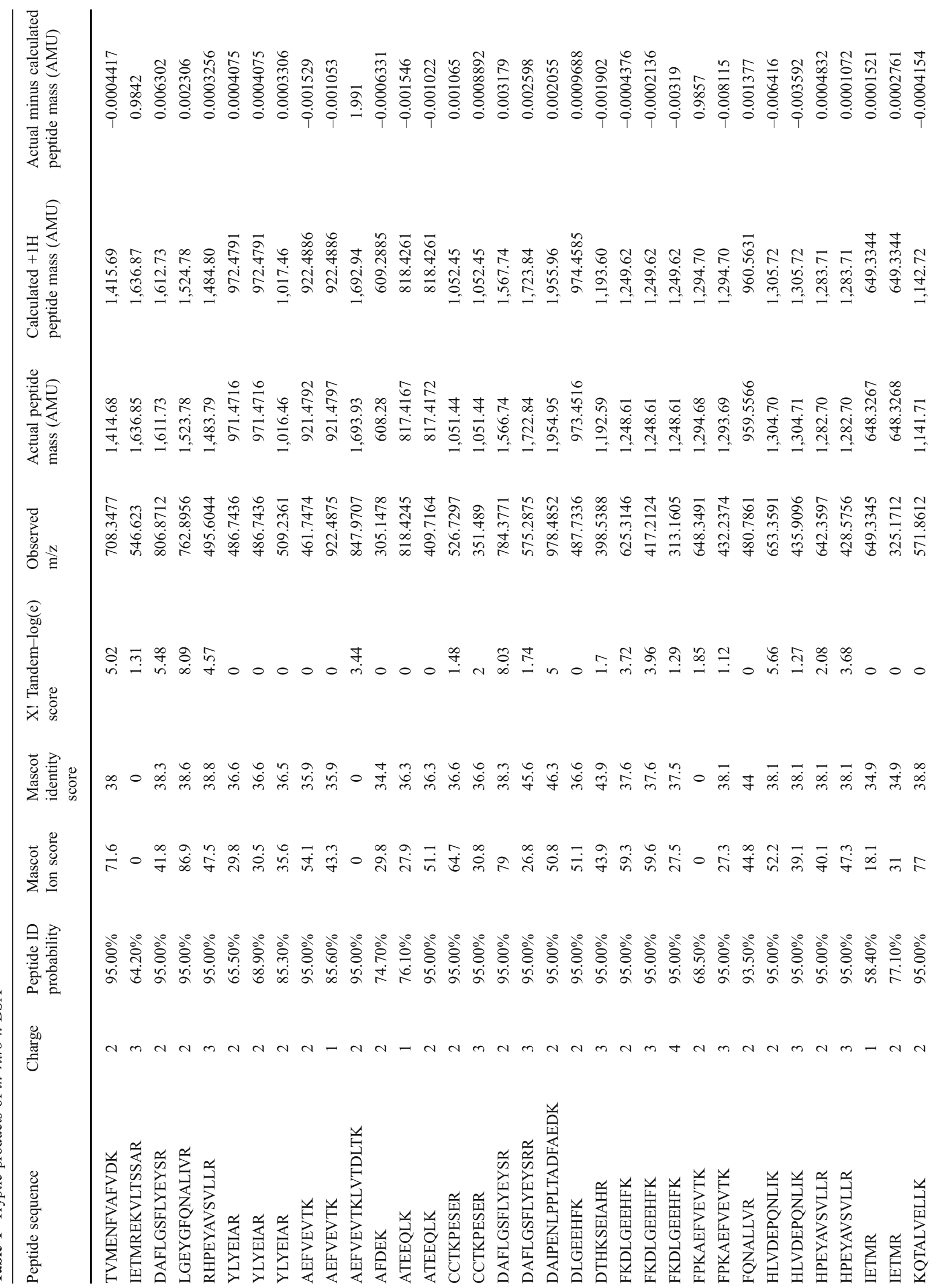




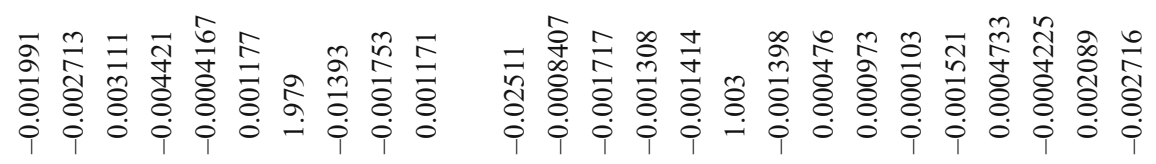

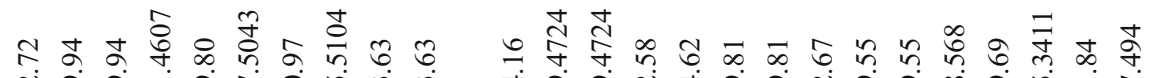
过

․

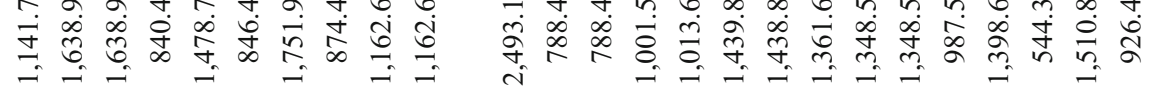

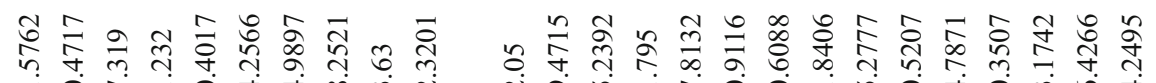

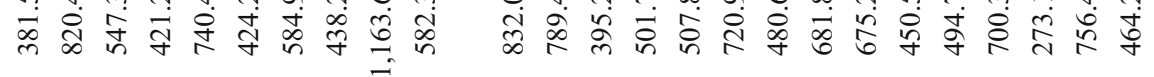

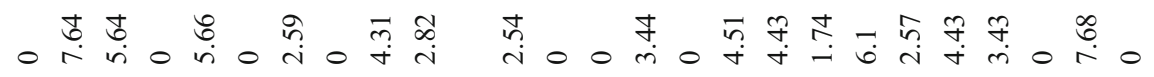

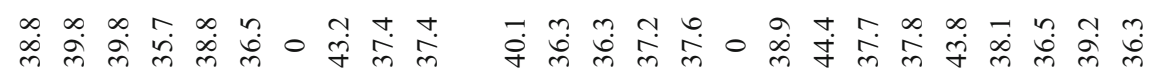

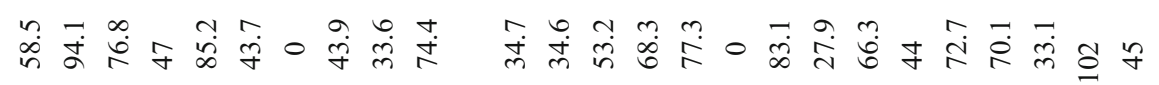

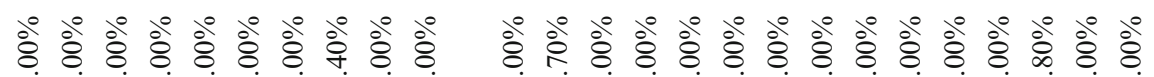
मूँ

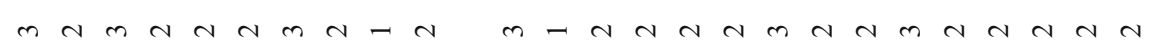

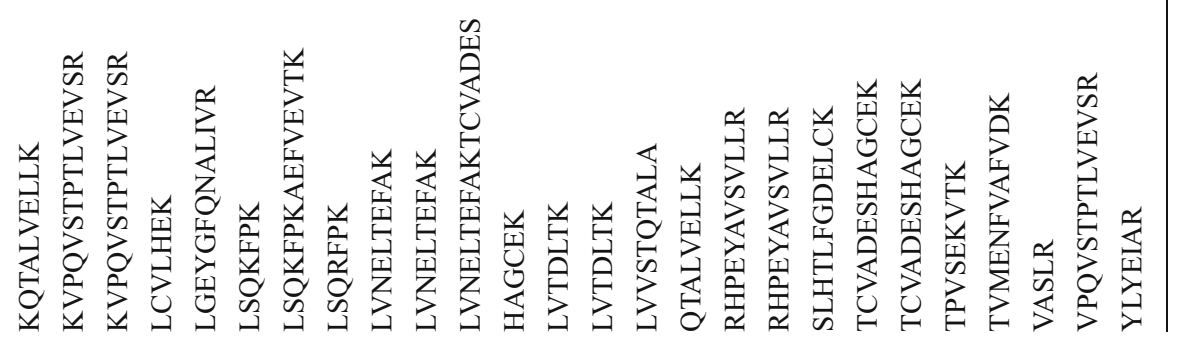


$5 \%(v / v)$ acetic acid in water for a minimum of $30 \mathrm{~min}$, followed by a second fixation step in $50 \% \mathrm{EtOH}$ for $10 \mathrm{~min}$. The gels were then washed twice for $10 \mathrm{~min}$ in water to remove the remaining acid. Prior to staining, the gels were sensitized for $2 \mathrm{~min}$ using an aqueous solution of $0.02 \%(w / v)$ sodium thiosulfate followed by three washes for $5 \mathrm{~min}$ each with water to remove the excess sodium thiosulfate. After rinsing, the gels were incubated for $30 \mathrm{~min}$ with gentle agitation in chilled $0.1 \%(w / v)$ silver nitrate. Following staining with silver nitrate, the gels were developed in $2 \%(w / v)$ sodium carbonate containing $0.037 \%(\mathrm{v} / \mathrm{v})$ formaldehyde in water. The staining reaction was quenched with an aqueous solution of $5 \%(v / v)$ acetic acid for $5 \mathrm{~min}$. The gels were stored at $4^{\circ} \mathrm{C}$ in $1 \%$ acetic acid in water until further analysis.

Immunoblot Analysis The nitrotyrosine and L-PGDS immunoprecipitation elution fractions were separated by SDS-
PAGE on a NuPAGE 4-12\% Bis-Tris gel (Invitrogen, Carlsbad, CA). The proteins were then transferred to a polyvinylidene fluoride membrane using the $\operatorname{iBlot}^{\mathrm{TM}} \mathrm{Gel}$ Transfer Device (Invitrogen, Carlsbad, CA) and the SNAP i.d. ${ }^{\mathrm{TM}}$ protein detection system (Millipore, Billerica, MA) was used to conduct the immunoblot. The primary antibodies used were mouse monoclonal anti-nitrotyrosine $(2 \mu \mathrm{g} / \mathrm{mL}$; Upstate, Lake Placid, NY) and rat monoclonal anti-L-PGDS ( $2 \mu \mathrm{g} / \mathrm{mL}$; Cayman Chemical, Ann Arbor, MI). The respective secondary antibodies (1:5,000 dilution) were goat anti-mouse IgG IRDye $^{\circledR} 680 \mathrm{~nm}$ (LI-COR Biosciences, Lincoln, NE) and goat anti-rat IgG IRDye ${ }^{\circledR} 680 \mathrm{~nm}$ (LI-COR Biosciences). The fluorescent blots were imaged on the Odyssey infrared imaging system (LI-COR Biosciences).

In-gel Trypsin Digestion Stained protein bands were excised and destained in $100 \mu \mathrm{L}$ of destaining solution (1:1
A) Normalized Ion Chromatograms

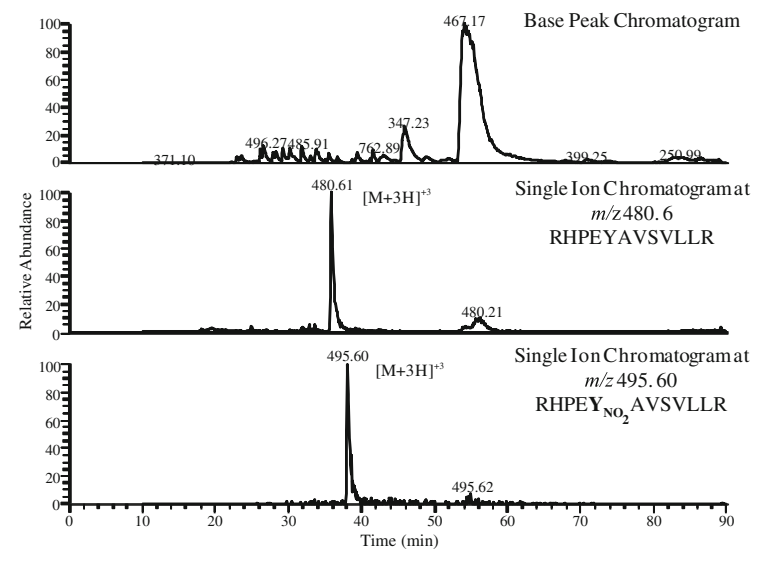

B) Tyrosine and Nitrotyrosine Immonium Ion Detection $\left([\mathrm{M}+3 \mathrm{H}]^{s} \mathrm{~m} / \mathrm{z} 175-190\right)$

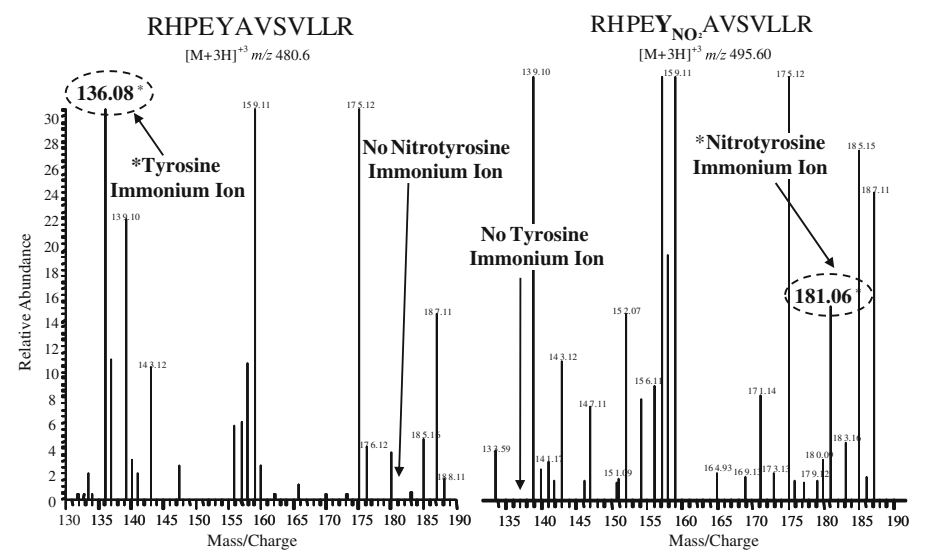

\section{C) Tandem MS of in vitron-BSA Peptide}

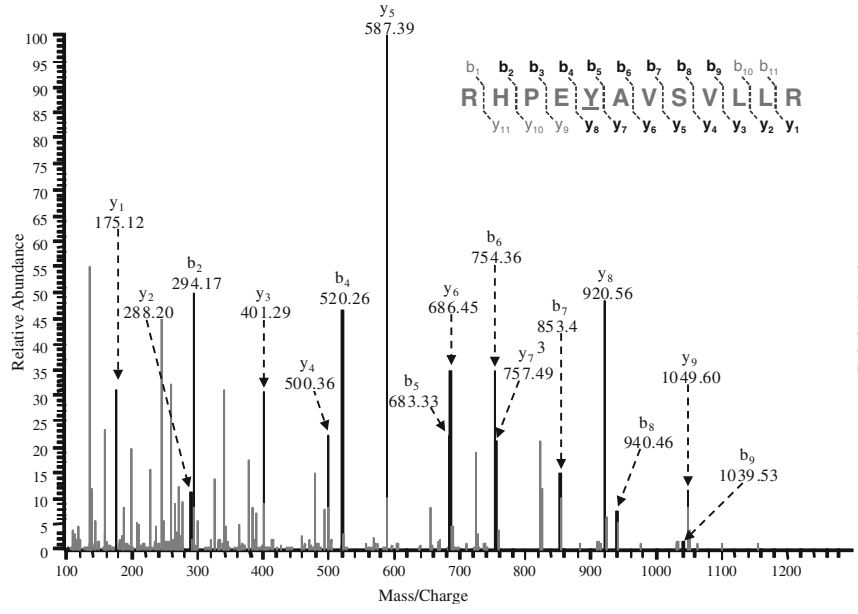

Fig. 3 LTQ-Orbitrap analysis of in vitro $n$-BSA tryptic peptides: RHPEYAVSVLLR and RHPEYNO2AVSVLLR. The base peak ion chromatograms of the MS analysis of the tryptic digestion of in vitro $n$-BSA are presented in Panel A. The single ion chromatograms at $m / z$ 480.61 and $m / z 495.60$ represent the detection of the $[\mathrm{M}+3 \mathrm{H}]+3$ ion of

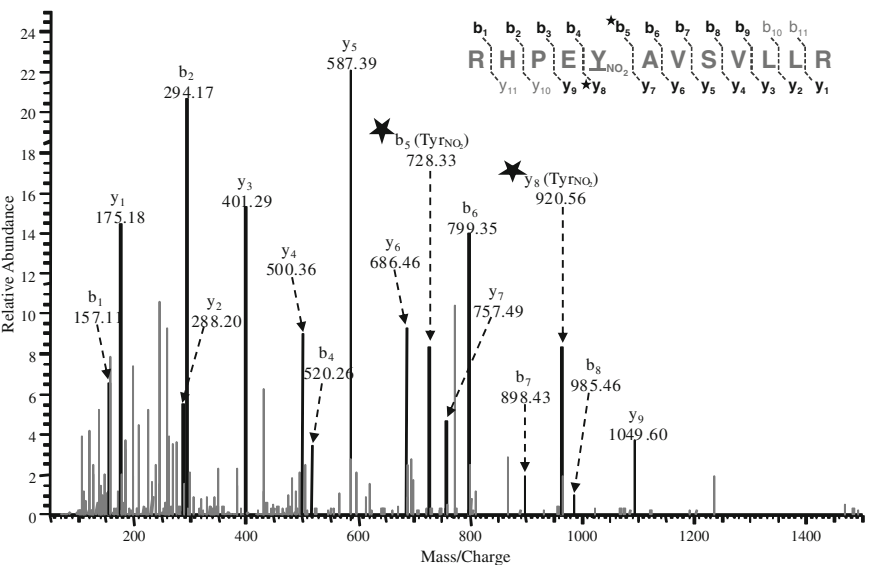

the non-nitrated and nitrated BSA peptide, respectively. The tandem MS spectra of $\mathrm{m} / \mathrm{z} 480.61$ and $\mathrm{m} / \mathrm{z} 495.60$ are in Panel C. In addition to changing the peptide mass, the $-\mathrm{NO} 2$ group also increases the Tyrosine immonium ion from $\mathrm{m} / \mathrm{z} 136.10$ to $\mathrm{m} / \mathrm{z} 181.06$ (Panel B) 
mixture of $30 \mathrm{mM}$ potassium ferricyanide: $100 \mathrm{mM}$ sodium thiosulfate) for $10 \mathrm{~min}$. at room temperature. The gel bands were washed with $100 \mathrm{mM}$ ammonium bicarbonate $\left(\mathrm{NH}_{4} \mathrm{HCO}_{3}\right)$ and dehydrated with ACN. After drying the bands in the speedvac, pre-selected gel bands were rehydrated in a trypsin solution (10 $\mathrm{ng}$ of trypsin in $50 \mathrm{mM} \mathrm{NH} \mathrm{HCO}_{3}$ ) on ice for $45 \mathrm{~min}$. The supernatant was removed and $100 \mu \mathrm{L}$ of $50 \mathrm{mM} \mathrm{NH}_{4} \mathrm{HCO}_{3}$ was added to the bands and the bands were placed at $37^{\circ} \mathrm{C}$ overnight for digestion. The supernatants were removed and the remaining peptides were extracted with $50 \mu \mathrm{L}$ of $1: 1$ mixture of $\mathrm{ACN}$ and $5 \%$ formic acid in water. Following extraction, the peptides were dried in a speedvac and resuspended in $20 \mu \mathrm{L}$ of LC-MS loading buffer $(3 \% \mathrm{ACN} /$ $0.1 \%$ formic acid in $\mathrm{H}_{2} \mathrm{O}$ ) for tandem MS analysis.

NanoLC LTQ-Orbitrap hybrid MS The peptide mixtures were separated by on-line reversed-phase (RP) nanoscale capillary liquid chromatography (nano-LC) and analyzed by ESI-tandem MS (LTQ-Orbitrap, Thermo Fisher Scien- tific, Berman, Germany). Prior to injection onto the reversed-phase column, the sample was injected onto a $10 \mu \mathrm{L}$ injection loop using the Agilent 1200 nanoflow autosampler (Agilent Technologies, Palo Alto, CA). From the injection loop, the sample was flushed onto the in-house packed fused-silica trap column at a flowrate of $5 \mu \mathrm{L} / \mathrm{min}$ to remove salt and particulate from the peptide mixture. After five washes, the 10-port valve switched from offline to inline with the mobile phase gradient.

For peptide separation, the peptide mixture was injected onto the $12 \mathrm{~cm}$ reversed-phase, fused-silica capillary column (ID: $75 \mu \mathrm{m}$, packed in-house with $\mathrm{C}_{18} 5 \mu \mathrm{m}$ packing material, YMC Europe $\mathrm{GmbH}$ ) using the Eksigent nanoLC-2D system (Eksigent, Dublin, CA). The aqueous mobile phase consisted of $0.1 \%$ formic acid in $\mathrm{H}_{2} \mathrm{O}$ (Solvent A) and the organic mobile phase consisted of $0.1 \%$ formic acid in ACN (Solvent B). The peptides were eluted from the hydrophobic stationary phase with a linear gradient and the total gradient time was 90 min. Solvent B was held constant at $3 \%$ for $5 \mathrm{~min}$, ramped to $10 \%$ in $5 \mathrm{~min}$
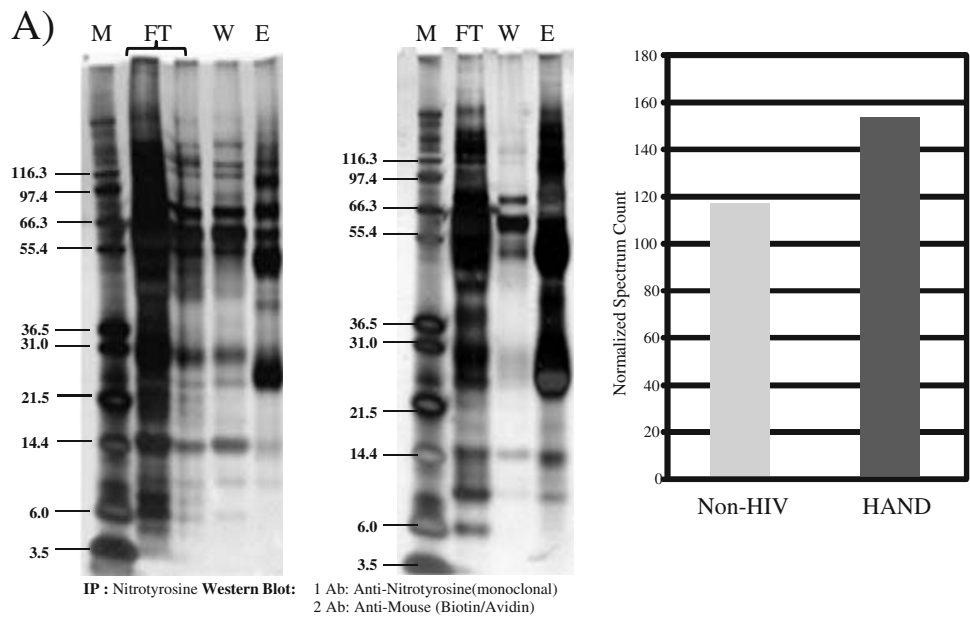

C)

Total L-PGDS
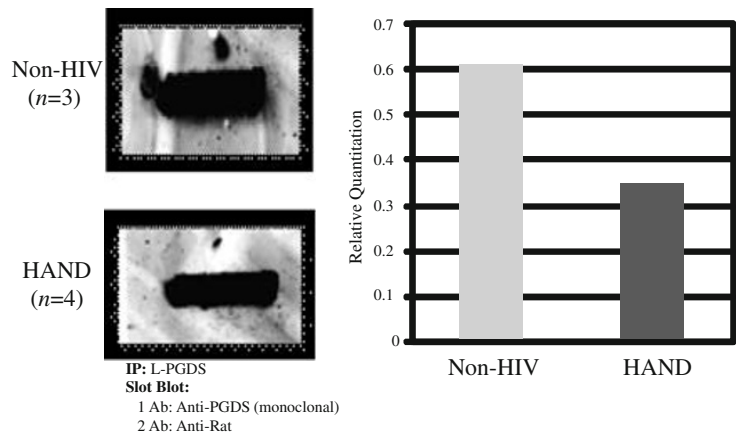

Fig. 4 1-D electrophoresis (a) of nitrotyrosine enrichment fractions from pooled non-HIV (control) and HAND CSF. Lane 1, MW marker; lane 2, flowthrough (non-nitrated proteins); lane 3, pre-elution PBS wash; and lane 4, 5\% formic acid elution (enriched nitrated proteins). The total number of spectra acquired (normalized spectrum count)
B) Total $\mathrm{NO}_{2}$-Modified CSF Protein
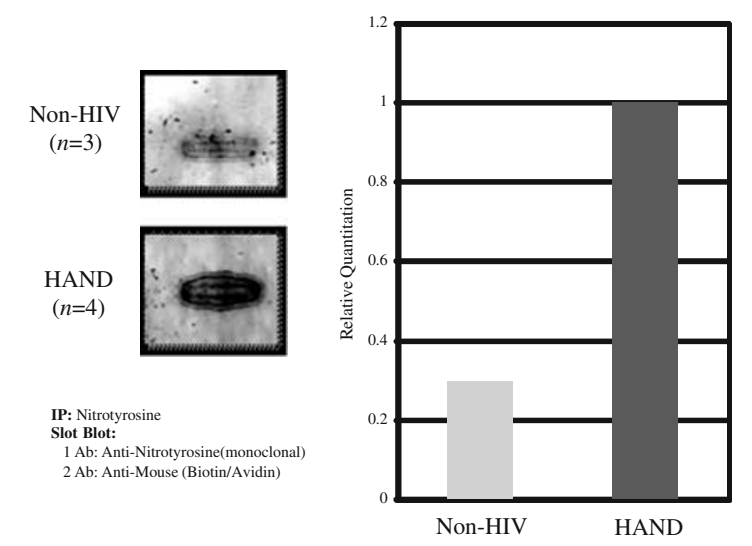

Total $\mathrm{NO}_{2}$-Modified L-PGDS
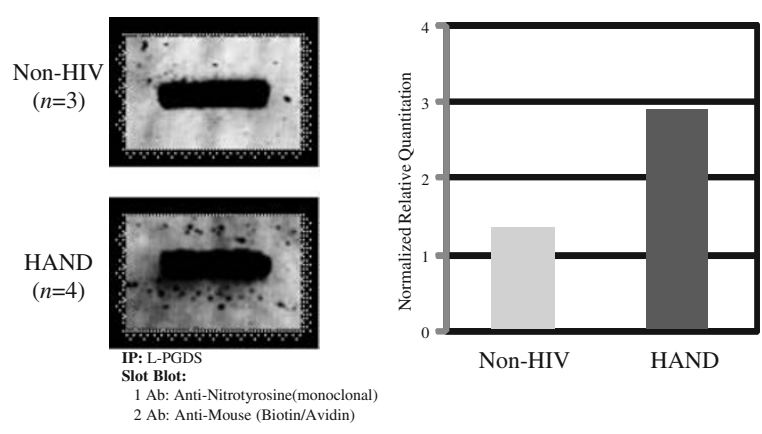

from Non-HIV (control) and HAND CSF nitrotyrosine-modified subproteome (a). Quantitative immunoblot analysis of nitrotyrosine content in albumin/IgG-depleted non-HIV (control) and HAND (diseased) CSF (b) and L-PGDS (c) determined by slot blot analysis 
and to $20 \%$ in $5 \mathrm{~min}$, followed by an increase to $60 \%$ at $60 \mathrm{~min}$. At $80 \mathrm{~min}$, Solvent B was increased to $97 \%$ and held constant for $2 \mathrm{~min}$ and ramped down to $3 \%$ at $85 \mathrm{~min}$ to re-equilibrate the reversed-phase column. The eluted peptides were directly transferred from the capillary column to a laser-pulled electrospray ionization emitter tip (New Objective, Woburn, MA) at a spray voltage of $2.0 \mathrm{kV}$. Datadependent acquisition was performed on the LTQ-Orbitrap (Thermo Fisher Scientific) mass spectrometer in the positiveion mode. The survey full MS scans $(m / z 200-2,000)$ were acquired in the Orbitrap mass analyzer with a resolution of $R=15,000$. The four most intense ions from the full MS scan were fragmented with collision-induced dissociation (CID) fragmentation and the MS/MS scans $(m / z 100-2,000)$ were also acquired in the Orbitrap with a resolution of $R=7,500$. The fragmented target ions were dynamically excluded for $90 \mathrm{~s}$. General mass spectrometric conditions were as follows: no sheath and auxiliary gas; ion transfer tube temperature of $200^{\circ} \mathrm{C}$; collision gas pressure was 1.5 mTorr; normalized collision energy was $35.0 \%$; ion selection threshold was 10,000 ; the activation q-value was 0.25 ; and the activation time was $30 \mathrm{~ms}$.

Database Search and Data Analysis The tandem mass spectra were extracted and the charge state deconvoluted by Mascot Distiller (v. 2.0, Matrix Science). De-isotoping was not performed. The MS/MS samples were analyzed using MASCOT (Matrix Science (version 2.2), London, UK) and $\mathrm{X}$ ! Tandem (www.thegpm.org; version 2007.01.01.1). The IPI_Human_20060712 protein database (selected for Homo sapiens, version 3.49, 60397 entries) was used by both MASCOT and X! Tandem to search the tandem MS data. For Orbitrap data, a 0.1 Da mass tolerance was allowed for parent ion masses and a $0.5 \mathrm{Da}$ mass tolerance was used for

Table 2 Relative quantification of nitrotyrosine-modified proteins from non-HIV and HAND CSF

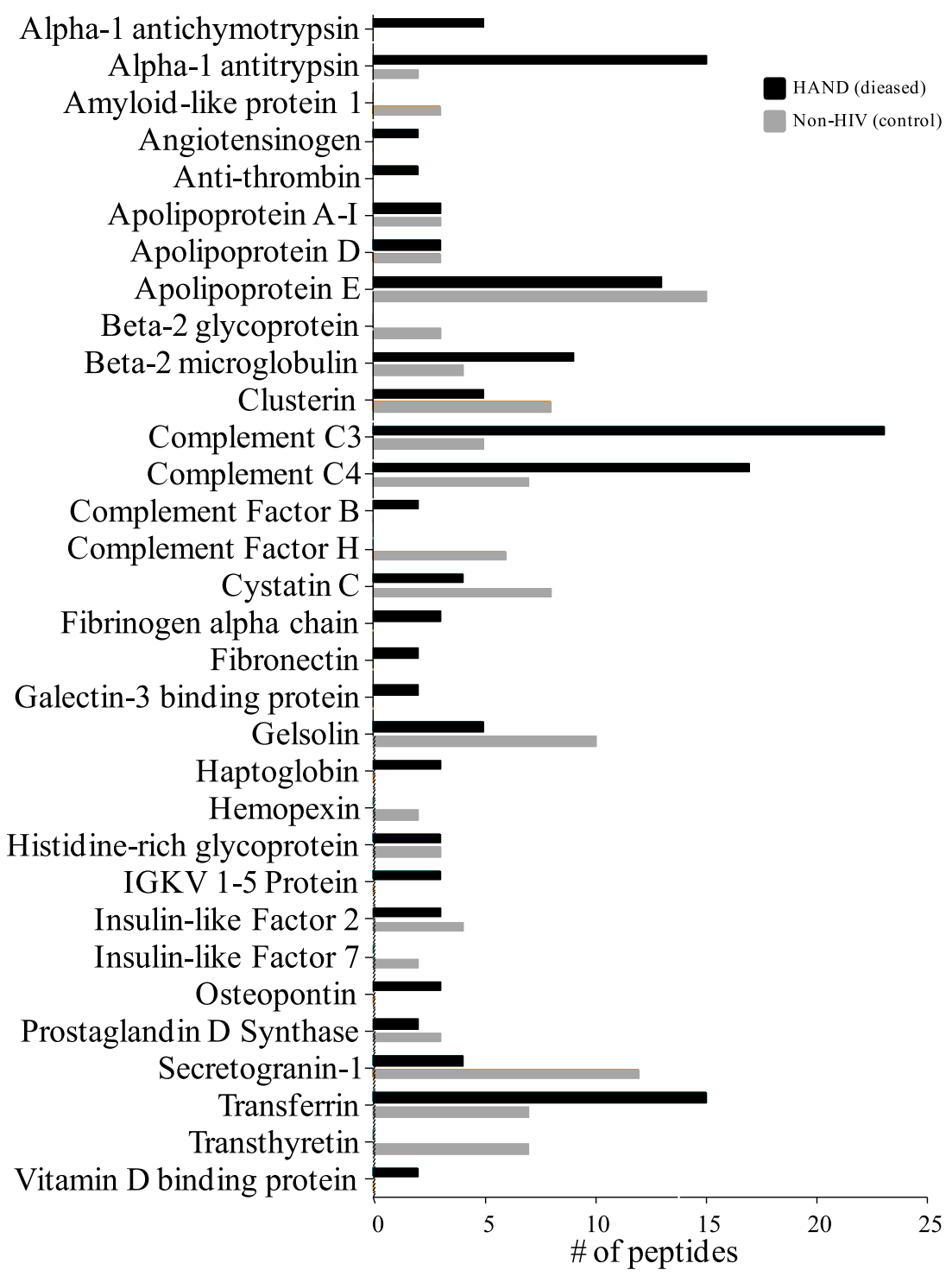


the product ions that were created via CID fragmentation. Two variable modifications were used for both MASCOT and X! Tandem: oxidation of methionine and nitration of tyrosine. The results from MASCOT and X! Tandem were uploaded onto the Scaffold Proteome software (Proteome Software Inc., Portland, OR) to formulate a final protein list. Scaffold Proteome software employs PeptideProphet and ProteinProphet algorithms to generate a bimodal histogram of the correct and incorrect identifications. The algorithms use the histogram to estimate the degree of overlap to determine the probability cutoff for correct peptide identifications. The Scaffold Proteome data sets were annotated and analyzed using ProteinCenter (Proxeon Biosystems, Odense Denmark).

\section{Results}

In vitro Nitrotyrosine Results: In vitro $n$-BSA Model To validate the affinity enrichment of in vitro nitrotyrosinemodified BSA, the antibody-enriched $n$-BSA was analyzed via reversed-phase HPLC (RP-HPLC). The RP-HPLC chromatogram in Fig. 2 displays a peak representative of in vitro $n$-BSA at 214 and $350 \mathrm{~nm}$. Unmodified angiotensin II was added as an internal standard and the angiotensin II peak was present only in the $214 \mathrm{~nm}$ chromatogram. The presence of in vitro $n$-BSA at both 214 and $350 \mathrm{~nm}$ validates in vitro nitration and affinity enrichment because the peptide bond absorbs at $214 \mathrm{~nm}$ and the $-\mathrm{NO}_{2}$ group attached to the tyrosine residue(s) absorbs at $350 \mathrm{~nm}$. The absorbance of unmodified tyrosine is $274 \mathrm{~nm}$; however, the presence of the $-\mathrm{NO}_{2}$ group alters the physical and chemical properties of the tyrosine residue, leading to a shift in UV absorption of tyrosine. Absorption of nitrated tyrosine is $\mathrm{pH}$-dependent, at acidic $\mathrm{pH}$ the prominent band of free nitrotyrosine was found to be at $350 \mathrm{~nm}$, which represents the neutral form of nitrotyrosine [10, 11]. However, at basic $\mathrm{pH}$ the major band of free nitrotyrosine was found to be at $422 \mathrm{~nm}$, which represents the ionized form of nitrotyrosine (Fig. 2 Inset) [10, 11]. Following RPHPLC analysis, the co-immunoprecipitation fractions were digested and analyzed by tandem MS via LTQ-Orbitrap. The peptides identified from tryptic digestion of in vitro $n$ BSA are summarized in Table 1, however, few nitrated peptides were observed in the analysis. This may be due to the differential nitration of tyrosine residues on BSA proteins and/or ion suppression during MS/MS analysis. The non-nitrated and nitrated RHPEYAVSVLLR ions were detected in the in vitro $n$-BSA digest (Fig. 3a-c). The detection of both the nitrated and non-nitrated forms of the peptide may stem from the differential nitration of the BSA protein. From the total (normalized) ion chromatogram, the triply charged ion for the non-nitrated $(\mathrm{m} / \mathrm{z} 480.61)$ and nitrated $(\mathrm{m} / \mathrm{z}$ 495.60) form of RHPEYAVSVLLR are present in the selected ion chromatograms (Fig. 3a). Due to the attachment of the $-\mathrm{NO}_{2}$ group on $\mathrm{Tyr}^{364}$, the peptides

Table 3 Identified peptides from tryptic digest of in vivo nitrated L-PGDS from non-HIV (control) and HAND CSF

\begin{tabular}{|c|c|c|c|c|c|c|}
\hline Peptide sequence & Charge & $\begin{array}{l}\text { Mascot Ion } \\
\text { Score }\end{array}$ & $\begin{array}{l}\text { Observed } \\
\mathrm{m} / \mathrm{z}\end{array}$ & $\begin{array}{l}\text { Actual } \\
\text { peptide mass } \\
\text { (AMU) }\end{array}$ & $\begin{array}{l}\text { Calculated }+1 \mathrm{H} \\
\text { peptide mass } \\
(\mathrm{AMU})\end{array}$ & $\begin{array}{l}\text { Actual minus } \\
\text { calculated peptide } \\
\text { mass (AMU) }\end{array}$ \\
\hline \multicolumn{7}{|l|}{ Non-HIV $(n=3)$} \\
\hline AQGFTEDTIVFLPQTDK & 2 & 54 & 955.5187 & $1,909.0228$ & $1,908.9469$ & 0.0759 \\
\hline EKFTAFCKAQGFTEDTIVFLPQTDK & 3 & 35 & 955.5182 & $2,863.5328$ & $2,863.4102$ & 0.1225 \\
\hline KNQCETRTM*LLQPAGSLGSYSYR & 3 & 30 & 873.8182 & $2,618.4328$ & $2,618.2581$ & 0.1747 \\
\hline SPHWGSTYSVSVVETDYDQYALLYS QGSK & 2 & 41 & $1,634.174$ & $3,266.3334$ & $3,266.5044$ & -0.171 \\
\hline SVVAPATDGGLNLTSTFLR & 2 & 132 & 960.0486 & $1,918.0826$ & $1,918.016$ & 0.0666 \\
\hline TM*LLQPAGSLGSYSYR & 2 & 68 & 881.0337 & $1,760.0528$ & $1,758.8611$ & 1.1918 \\
\hline WFSAGLASNSSWLR & 2 & 20 & 791.1036 & $1,580.1926$ & $1,580.7736$ & -0.5809 \\
\hline \multicolumn{7}{|l|}{ HAND $(n=4)$} \\
\hline AQGFTEDTIVFLPQTDK & 2 & 77 & 955.4286 & $1,908.8426$ & $1,908.9469$ & -0.1043 \\
\hline EKFTAFCKAQGFTEDTIVFLPQTDK & 3 & 42 & 955.4282 & $2,863.2628$ & $2,863.4102$ & -0.1475 \\
\hline KNQCETRTM*LLQPAGSLGSYSYR & 3 & 17 & 874.1215 & $2,619.3427$ & $2,618.2581$ & 1.0846 \\
\hline SPHWGSTYSVSVVETDYDQYALLYS QGSK & 3 & 74 & $1,090.261$ & $3,267.7612$ & $3,266.5044$ & 1.2568 \\
\hline SVVAPATDGGLNLTSTFLR & 2 & 129 & 959.8787 & $1,917.7428$ & $1,918.016$ & -0.2732 \\
\hline TMLLQPAGSLGSYSYR & 2 & 92 & 872.3737 & $1,742.7328$ & $1,742.8661$ & -0.1333 \\
\hline TMLLQPAGSLGSY*SYR & 2 & 50 & 894.941 & $1,787.88$ & $1,788.874$ & -0.994 \\
\hline WFSAGLASNSSWLR & 2 & 20 & 791.4687 & $1,580.9228$ & $1,580.7736$ & 0.1493 \\
\hline
\end{tabular}

$\mathrm{M}^{*}$ : oxidized methionine; $\mathrm{Y}^{*}: \mathrm{NO}_{2}$-modified tyrosine 
A) Normalized Ion Chromatograms

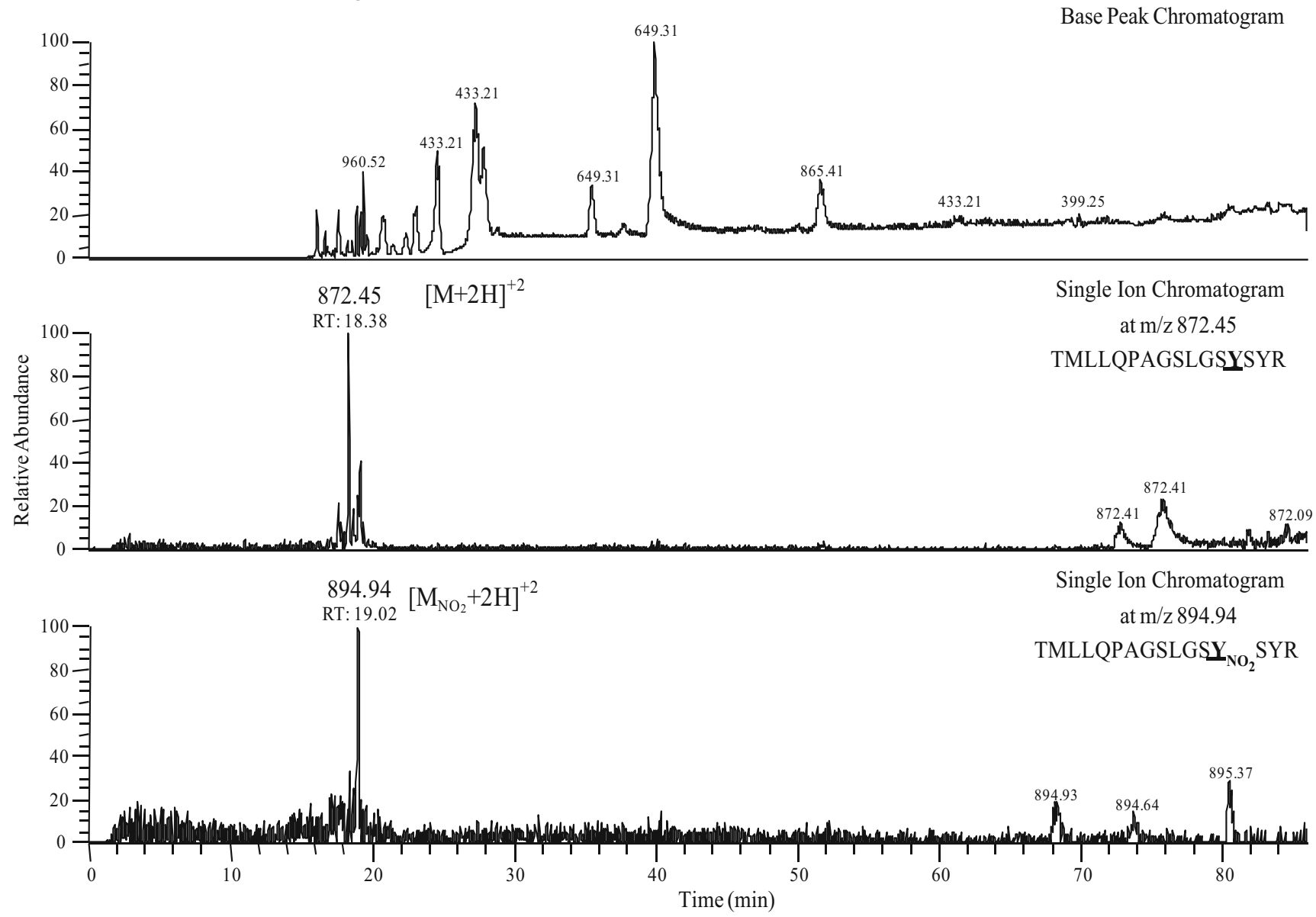

Fig. 5 LTQ-Orbitrap analysis of HAND L-PGDS tryptic peptides: TMLLQPAGSLGSYSYR and TMLLQPAGSLGSYNO2SYR. The base peak ion chromatograms of the MS analysis of the tryptic digestion of HAND L-PGDS are presented in Panel A. The single ion

elute within $\sim 1.0$ min of each other. The product ion spectrum for both forms contain relative complete series of $\mathrm{b}$ - and $\mathrm{y}$-ions and the difference between $\mathrm{b}_{5} / \mathrm{y}_{8}$ and $* \mathrm{~b}_{5} /{ }^{*} \mathrm{y}_{8}$ fragment ions (asterisks $(*)$ indicate nitrated spectrum) represent the location of the nitrotyrosine modification (Fig. 3c). The nitrotyrosine modification also shifts the immonium ion of tyrosine from $\mathrm{m} / \mathrm{z} 136.10$ to $\mathrm{m} / \mathrm{z} 181.06$, as observed in Fig. 3b. The shift in the $\mathrm{m} / z$ value of the tyrosine immonium ion offers an alternative route to distinguish nitrated peptides from non-nitrated peptides.

\section{In Vivo Nitrotyrosine Results}

Nitrotyrosine Sub-proteome of non-HIV and HAND CSF Following albumin/IgG depletion of the pooled CSF samples, slot blot analysis was used to determine the nitrotyrosine content in each sample. We confirmed a higher nitrotyrosine content in the pooled HAND CSF sample compared to the nitrotyrosine present in the non-HIV infected CSF (Fig. 4b). chromatograms at $\mathrm{m} / \mathrm{z} 872.48$ and $\mathrm{m} / \mathrm{z} 894.94$ represent the detection of the $[\mathrm{M}+2 \mathrm{H}]+2$ ion of the non-nitrated and nitrated HAND L-PGDS peptide, respectively. The tandem MS spectra of $\mathrm{m} / \mathrm{z} 872.48$ and $\mathrm{m} / \mathrm{z}$ 894.94 are in Panel B

The one-dimensional silver stained images in Fig. 4a represent the fractions from the affinity-based enrichment of both non-HIV and HAND CSF. From tandem MS analysis of the nitrotyrosine-modified tryptic digest products, a total of 32 proteins were identified (Electronic Supplementary Material, Table 1). Of the identified nitrated proteins, nine were unique to non-HIV CSF; eight were unique to HAND CSF; and 15 were observed in both CSF specimens. It is possible that the unique proteins identified in the non-HIV CSF are unique to the underlying disease state of these individuals. Alternatively, since the CSF from HIV-infected individuals were selected for high concentration of nitrated proteins it is possible that these proteins may have masked the other nitrated proteins present in low concentrations. The Scaffold Proteome software was used to evaluate the MS/MS data of the identified proteins from both groups and spectral counting was used as a pseudo-quantification method to determine differential expression of the nitrated proteins. As illustrated in Fig. 4a, there was an increase in the number of 
B) Tandem MS of in vivo nitrated L-PGDS Peptide
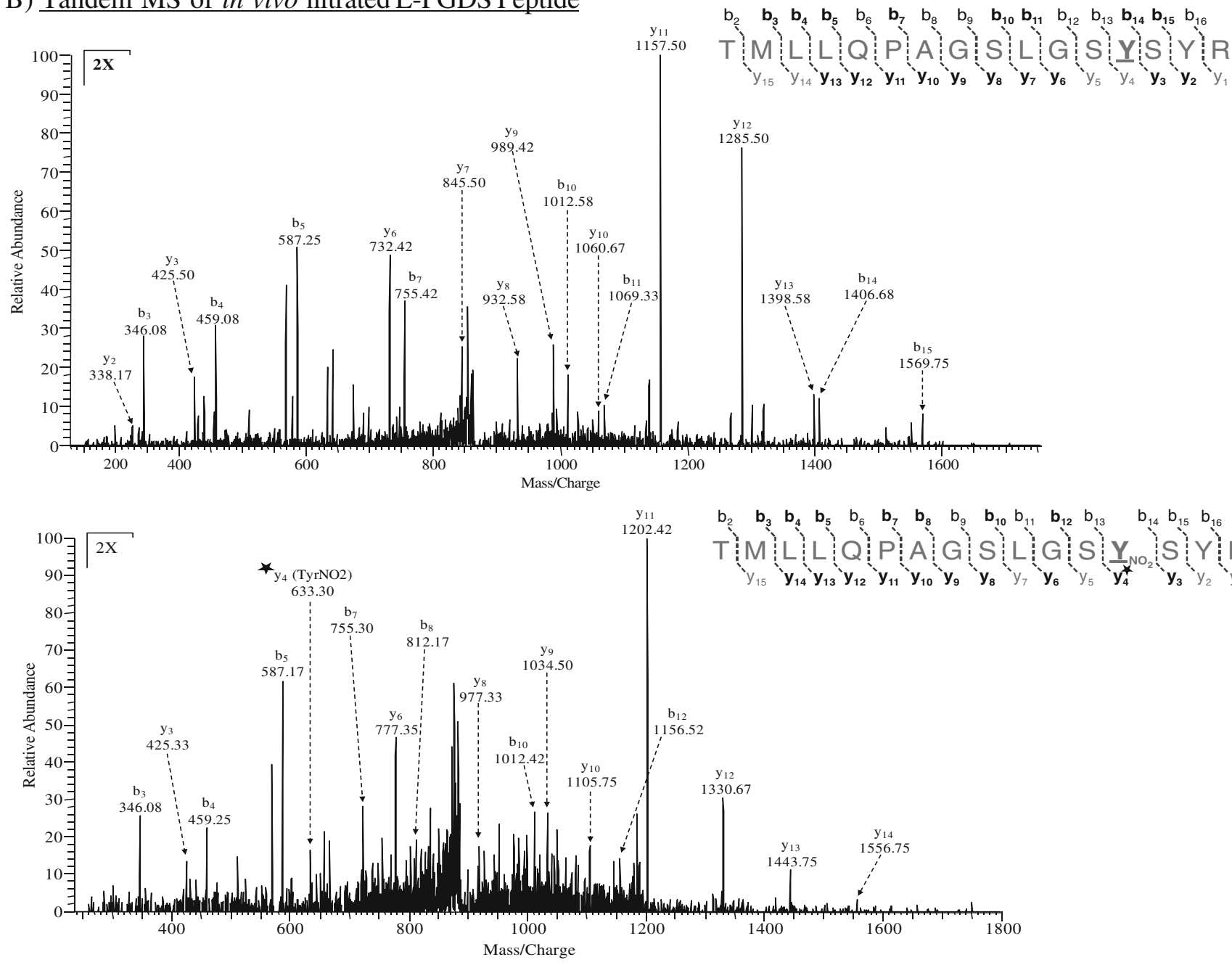

Fig. 5 (continued)

tandem MS spectra observed in the HAND CSF sample when compared to the spectra detected from the non-HIV infected CSF. This suggests that there was a higher concentration of nitrotyrosine-modified proteins in the HAND sample, compared to the non-HIV sample, which is consistent with the slot blot analysis. The bar graph in Table 2 represents the total number of peptides identified per protein in the non-HIV and HAND samples.

In Vivo L-PGDS Analysis from non-HIV and HAND CSF L-PGDS, also known as $\beta$-trace protein, is one of the most abundant brain-derived proteins in human CSF and is primarily expressed in the arachnoid membrane, leptomeninges, and choroid plexus [12]. In addition to expression in the central nervous system, L-PGDS is also expressed in male genital organs, heart, and bodily fluids (plasma, seminal fluid, amniotic fluid, CSF, and urine) [12]. LPGDS catalyzes the isomerization of prostaglandin- $\mathrm{H}_{2}$, the precursor to various prostanoids, to prostaglandin- $\mathrm{D}_{2}$ in the presence of sulfhydryl compounds and also functions as a transporter of small non-substrate lipophilic molecules, including thyroid hormone, retinoic acid, retinaldehyde, bilirubin, and biliverdin [12]. Using the Catch and Release $^{\mathrm{TM}}$ system, we were able to isolate nitrotyrosinemodified and unmodified L-PDGS from non-HIV and HAND CSF. The total L-PGDS and nitrotyrosine content from each sample was determined by density analysis of the slot blot images (Fig. 4c). The total L-PGDS content was higher in non-HIV CSF; however the content of in vivo nitrotyrosine-modified L-PGDS was substantially higher in HAND compared to non-HIV. This is possibly due to the increase in nitro-oxidative stress observed in HAND. These findings, however, need to be confirmed in individual samples with larger sample sizes with and without HAND.

In addition to quantitative immunoblot analysis, the enriched L-PGDS was digested with trypsin and analyzed by tandem MS (LTQ-Orbitrap). The peptides identified from each group are presented in Table 3 . We did not identify any 
nitrated peptides in non-HIV samples; however, the doubly charged non-nitrated $(\mathrm{m} / \mathrm{z} 872.44)$ and nitrated $(\mathrm{m} / \mathrm{z}$ 894.94) TMLLQPAGSLGSYSYR ion was identified in HAND CSF (Fig. 5a). Tandem MS analysis of modified (Fig. 5c) and unmodified (Fig. 5b) TMLLQPAGSLGSY $\left(\mathrm{NO}_{2}\right) \mathrm{SYR}$ revealed the presence of the $-\mathrm{NO}_{2}$ modification at $\mathrm{Tyr}^{105}$ $\left({ }^{*} \mathrm{y}_{4}\right.$-ion).

A previous study demonstrated that nitrotyrosine-modified L-PGDS exhibited a loss of enzymatic activity [12]. In this study L-PGDS was in vitro nitrated, which may have lead to extensive nitration of the molecule. However, from our current findings it appears that L-PGDS in CSF of individuals with HAND is nitrated at $\mathrm{Tyr}^{105}$, which is involved in transportation of small lipophilic molecules. $\mathrm{Tyr}^{105}$ is a highly conserved residue among members of the lipocalin family and is located in the hydrophobic aromatic cluster of the $\beta$-sheet barrel, which is thought to function as the ligand-binding pocket [13]. Structural studies of recombinant mouse L-PGDS reveal that the hydrophobic aromatic cluster plays a major role in the stabilization of retinoic acid [13]. Retinoic acid is an active metabolite of vitamin A and functions as a potent regulator of gene transcription. The stabilization of retinoic acid in the hydrophobic aromatic cluster stems from the relatively strong Van der Waals interactions between the isoprenoid chain and $\beta$-ionone ring of retinoic acid and the aromatic ring of Tyr $\left(\mathrm{Tyr}^{105}\right.$ and $\mathrm{Tyr}^{149}$ ) and Phe $\left(\mathrm{Phe}^{34}, \mathrm{Phe}^{39}\right)$ residues [13]. Nitration of $\mathrm{Tyr}^{105}$ alters the physical and chemical characteristics of the residue, including non-covalent and covalent interactions. It has been shown that the addition of the bulky $-\mathrm{NO}_{2}$ group reduces the pKa of the hydroxyl group of Tyr and also introduces steric restrictions to the phenolic ring of Tyr [6].

Therefore, we suggest that nitration of $\mathrm{Tyr}^{105}$ disrupts the stabilization of retinoic acid in the hydrophobic binding pocket of L-PGDS. Since L-PGDS is a major component of CSF and highly concentrated in the choroid plexus, which forms the barrier between blood and CSF, it is thought that L-PGDS facilitates the transport of the lipophilic small molecules (e.g., retinoic acid) across the blood/brain barrier [14]. Consequently, interference of the stabilization of retinoic acid as a result of $\mathrm{Tyr}^{105}$ nitration in the hydrophobic binding pocket of L-PGDS, could prevent the delivery of retinoic acid to specific target tissues. Previous studies have linked retinoid compounds to the suppression of proinflammatory cytokine and chemokine production during HIV-induced CNS inflammation [15]. Vitamin A deficiency has been associated with enhanced $\mathrm{T}$ cell-mediated proinflammatory immune response, which has been shown to contribute to immune-induced tissue damage and also the progression of human disease [15]. It is thought that the HIV infection triggers events that lead to a reduction in plasma levels of vitamin $\mathrm{A}$, which could increase the clinical consequences of HIV infection [15].
Therefore, the inability of nitrotyrosine-modified LPGDS to transport retinoic acid could potentially lead to the increased progression of the proinflammatory response of HIV-associated neurocognitive dysfunction.

\section{Conclusion}

Utilizing immunoaffinity-based enrichment and tandem mass spectrometry we were able to identify and characterize nitrotyrosine-modified proteins from HAND CSF. More specifically, we were able to isolate nitrotyrosine-modified LPGDS from CSF and characterize the modification site. We conclude from previous structures of mouse L-PGDS, that nitration of $\mathrm{Tyr}^{105}$ of L-PGDS can potentially alter its ability to transport retinoic acid, a known regulator of gene expression. A deficiency in vitamin A metabolites has been shown to contribute to the proinflammatory response observed during host invasion (e.g., HIV infection). In addition to its ligand-binding function, L-PGDS also converts prostaglandin- $\mathrm{H}_{2}$ into prostaglandin- $\mathrm{D}_{2}$ which is known to play a major role in the inflammatory response, sleep, and also vasodilation. Previous studies have observed elevated levels of prostaglandins in individuals diagnosed with HIVassociated neurocognitive dysfunction [16]. Therefore, further characterization of the influence of nitration on the function of L-PGDS in individuals diagnosed with HAND may lead to the development of potential clinical diagnostics or therapies for HIV-associated neurocognitive dysfunction.

Acknowledgments This work was supported by grants P30 MH075673 (JM) and P20 DA026164 (RJC) from the National Institutes of Health.

\section{References}

1. Ghafouri M, Amini S, Khalili K, Sawaya BE. HIV-1 associated dementia: symptoms and causes. Retrovirology. 2006;3:28.

2. Antinori A, Arendt G, Becker JT, Brew BJ, Byrd DA, Cherner M, et al. Updated research nosology for HIV-associated neurocognitive disorders. Neurology. 2007;69(18):1789-99.

3. Ances BM, Ellis RJ. Dementia and neurocognitive disorders due to HIV-1 infection. Semin Neurol. 2007;27(1):86-92.

4. Adamson DC, Wildemann B, Sasaki M, Glass JD, et al. Immunologic NO synthase: elevation in severe AIDS dementia and induction by HIV-1 gp41. Science. 1996;274:1917-20.

5. Ryberg H, Caidahl K. Chromatographic and mass spectrometric methods for quantitative determination of 3-nitrotyrosine in biological samples and their application to human samples. J Chromatogr B Analyt Technol Biomed Life Sci. 2007;851(1-2):160-71.

6. Radi R. Nitric oxide, oxidants, and protein tyrosine nitration. Proc Natl Acad Sci USA. 2004;101:4003-8.

7. Blum H, Beier H, Gross HJ. Improved silver staining of plant proteins. RNA and DNA in polyacrylamide gels. Electrophoresis. 1987;8:93-9.

8. Gharib M, Marcantonio M, Lehmann SG, Courcelles M, et al. Artifactual sulfation of silver-stained proteins: implications for the 
assignment of phosphorylation and sulfation sites. Mol Cell Proteomics. 2009;8(3):506-18.

9. Riordan JF, Sokolovsky M, Vallee BL. Environmentally sensitive tyrosyl residues. Nitration with tetranitomethane. Biochemistry. 1967;6:358-61.

10. Dr Filippis V, Frasson R, Fontana A. 3-Nitrotyrosine as a spectroscopic probe for investigating protein-protein interaction. Protein Sci. 2006;15(5):976-86.

11. Lescuyer P, Gandini A, Burkhar PR, Hochstrasser DF, et al. Prostaglandin D2 synthase and its post-translational modifications in neurological disorders. Electrophoresis. 2005;26(23):4563-70.

12. Li W, Malpica-Llanos TM, Gundry R, Cotter RJ, et al. Nitrosative stress with HIV dementia causes decreased L-prostaglandin D synthase activity. Neurology. 2008;70(19 Pt 2):1753-62.
13. Urade Y, Hayaishi O. Biochemical, structural, genetic, physiological, and pathophysiological features of lipocalin-type prostaglandin D synthase. Biochim Biophys Acta. 2000;1482(1-2): 259-71.

14. Flower D. The lipocalin protein family: structure and function. Biochem J. 1996;318:1-14.

15. Royal III W, Vlahov D, Lyles C, Gajewski CD, et al. Retinoids and drugs of abuse: implications for neurological disease risk in human immunodeficiency virus type 1 infection. Clin Infect Dis. 2003;37 Suppl 5:S427-32.

16. Shimamoto $\mathrm{S}$, Yoshida $\mathrm{T}$, Inui $\mathrm{T}$, Gohda $\mathrm{K}$, et al. NMR structure of lipocalin-type prostaglandin $\mathrm{D}$ synthase: evidence for partial overlapping of catalytic pocket and retinoic acid-binding pocket within the central cavity. J Biol Chem. 2007;282(43):31373-9. 